\title{
GEOMETRIC VERSUS HOMOTOPY THEORETIC EQUIVARIANT BORDISM
}

\author{
BERNHARD HANKE
}

\begin{abstract}
By results of Löffler and Comezaña, the Pontrjagin-Thom map from geometric $G$-equivariant bordism to homotopy theoretic equivariant bordism is injective for compact abelian $G$. If $G=S^{1} \times \ldots \times S^{1}$, we prove that the associated fixed point square is a pull back square, thus confirming a recent conjecture of Sinha [22]. This is used in order to determine the image of the Pontrjagin-Thom map for toral $G$.
\end{abstract}

\section{INTRODUCTION}

Geometric equivariant bordism theory was introduced by Conner and Floyd [7] as an important tool for studying groups of transformations on smooth manifolds. Later, the homotopy theoretic analogue was described by tom Dieck [8] and in a slightly different way by Bröcker and Hook [2] in order to develop a conceptual approach to the localization theorem of Atiyah-Segal [1] and to study equivariant characteristic numbers of $G$ manifolds [9, 10]. The homotopy theoretic definition turned out to be the correct description of equivariant bordism in the context of equivariant stable homotopy theory, because it is stable under suspensions with arbitrary $G$-representations. It has gained recent interest in the context of general completion theorems [13] analogous to the Atiyah-Segal completion theorem in equivariant $K$-theory and in connection with the study of equivariant formal group laws [4, 5].

The coefficients of equivariant bordism theories are difficult to calculate and only partial results are known. Generators for the geometric equivariant bordism rings have been found in [16] for $G=S^{1}$ and in [15] for $G=\mathbb{Z} / p$. The semifree geometric $S^{1}$-bordism ring is calculated in [22]. In [17], the homotopy theoretic $\mathbb{Z} / p$-bordism ring is described in terms of a pull back square involving localization with respect to nontrivial Euler classes. The paper [21] investigates generators and relations in the homotopy theoretic $S^{1}$-bordism ring.

Let $G$ be a compact Lie group. We denote by $\Omega_{*}^{G}$ the geometric unitary $G$-bordism ring and by $M U_{*}^{G}$ the homotopy theoretic unitary $G$-bordism ring (for definitions see Section 2 below). Both theories are regarded as graded over the integers. 
Similar to the nonequivariant case there is a Pontrjagin-Thom map

$$
\Psi: \Omega_{*}^{G} \rightarrow M U_{*}^{G} .
$$

However, the proof of $\Psi$ being an isomorphism fails if $G \neq\{e\}$ due to the lack of generic equivariant transversality [20].

If $G=\mathbb{Z} / p$ is of prime order, tom Dieck [8] showed that $\Psi$ is injective. This result was generalized by Löffler [19] and Comezaña [6] to the case that $G$ is a compact abelian Lie group. It is not known, if $\Psi$ is injective in general.

On the other hand, if $G$ is nontrivial, then $M U_{*}^{G}$ contains nonzero elements in negative degrees: Each complex $G$-representation $W$ gives rise to an Euler class

$$
e_{W} \in M U_{-2|W|}^{G}
$$

which is zero if and only if $W^{G} \neq 0$. Here and in the following $|W|$ denotes the complex dimension of a complex $G$-representation $W$. In particular, $\Psi$ is not surjective, if $G \neq\{e\}$, since the geometrically defined theory $\Omega_{*}^{G}$ is concentrated in nonnegative degrees.

Our paper contributes to the understanding of the relation between geometric and homotopy theoretic equivariant bordism if $G$ is a torus of the form $S^{1} \times \ldots \times S^{1}$. On the one hand it complements the classical work of tom Dieck, Löffler and others. On the other hand it should provide some useful information in connection with the present interest in equivariant stable homotopy theory.

In the recent paper [22] the image of $\Psi$ is described for semifree $S^{1}$ bordism and $\mathbb{Z} / p$-bordism. Conjecture 4.1 . in loc. cit. gives a conjectural description of $\operatorname{im}(\Psi)$ for $G=S^{1}$.

For toral $G$, in Theorem 1 we show the existence of a pull back square

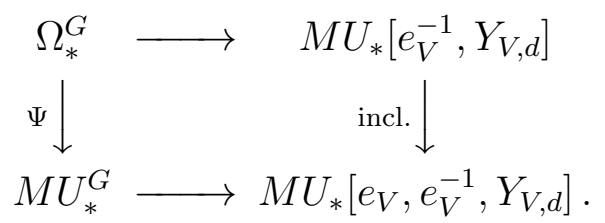

It refines [8], Proposition 4.1, and generalizes [22], Corollary 2.11. In particular, it confirms the aforementioned conjecture. The horizontal maps in this diagram capture the normal data around fixed point sets of elements in $\Omega_{*}^{G}$ and $M U_{*}^{G}$. These normal data are expressed in terms of polynomials with $M U_{*}$-coefficients in Euler classes $e_{V}$ of irreducible complex $G$ representations ( $V$ running through a complete set of isomorphism classes of nontrivial irreducible complex $G$-representations), their formal inverses and in certain classes $Y_{V, d}, 2 \leq d<\infty$, of degree $2 d$. We can think of $e_{V}^{-1}$ 
as being geometrically represented by the $G$-bundle

$$
V \rightarrow *
$$

and - more generally - of the classes $Y_{V, d}$ as being represented by the $G$ bundles

$$
E \otimes V \rightarrow \mathbb{C} P^{d-1}
$$

where $E \rightarrow \mathbb{C} P^{d-1}$ is the hyperplane line bundle (i.e. the normal bundle of $\mathbb{C} P^{d-1}$ in $\left.\mathbb{C} P^{d}\right)$.

The horizontal maps in the above diagram are injective (assuming that $G$ is a torus) and we can conclude that the Pontrjagin-Thom map induces a ring isomorphism

$$
\Omega_{*}^{G} \cong M U_{*}^{G} \cap M U_{*}\left[e_{V}^{-1}, Y_{V, d}\right],
$$

this intersection taking place in $M U_{*}\left[e_{V}, e_{V}^{-1}, Y_{V, d}\right]$. In particular, the realizability of an element in $M U_{*}^{G}$ as an actual $G$-manifold is expressed in terms of a representation theoretic condition on the normal data around the fixed point set. This should be contrasted with the usual failure of $G$-equivariant transversality as discussed in [20]: In general $G$-equivariant transversality is obstructed not only by local invariants expressible in terms of Euler classes, but also by global ones, see Theorem 1 in loc. cit. In the context of equivariant bordism however, the vanishing of the local obstructions turns out to be sufficient for equivariant transversality within the equivariant homotopy class of a given stable map $S^{W} \rightarrow T\left(\xi_{n}^{G}\right)$ with respect to the zero section of $T\left(\xi_{n}^{G}\right)$. Here, $T\left(\xi_{n}^{G}\right)$ denotes a certain equivariant Thom space (see below) and $S^{W}$ is the one point compactification $W \cup\{\infty\}$ with base point $\infty$.

If $G$ is not a torus and not of prime order, geometric realizability can not be read off from normal data around the fixed point set. We will illustrate this by easy examples.

In the case of toral $G$, we refine the description of geometric $G$-bordism using the fact that the image of the fixed point map

$$
M U_{*}^{G} \rightarrow M U_{*}\left[e_{V}, e_{V}^{-1}, Y_{V, d}\right]
$$

is characterized by integrality conditions [8]. Details will be given below.

The methods used in the proof of Theorem 1 are mainly classical, based on considering bordism with respect to families of subgroups and equivariant characteristic numbers.

\section{REVIEW OF EQUIVARIANT BORDISM}

We briefly recall the definitions and constructions that are necessary in order to formulate our results. If not stated otherwise, $G$ denotes a compact Lie group. 
Definition 1. Let $M$ be a closed smooth $G$-manifold. We define a stable almost complex $G$-structure on $M$ to be a complex $G$-structure on

$$
T M \oplus \underline{\mathbb{R}}^{k} \rightarrow M
$$

for some $k$. Here, the trivial bundle

$$
\underline{\mathbb{R}}^{k}=M \times \mathbb{R}^{k} \rightarrow M
$$

is equipped with the trivial $G$-action on the fibres $\mathbb{R}^{k}$ and by a complex $G$-structure, we mean a $G$-equivariant bundle map

$$
J: T M \oplus \underline{\mathbb{R}}^{k} \rightarrow T M \oplus \underline{\mathbb{R}}^{k}
$$

over $M$ which satisfies $J^{2}=-1$. Two stable almost complex $G$-structures are identified, if after stabilization with further $\mathbb{C}$-summands (equipped with trivial $G$-actions on the fibres), the induced complex $G$-structures are $G$ homotopic through complex $G$-structures.

Note that with this definition, the (unstabilized) normal bundle of the $G$ embedding

$$
M^{G} \subset M
$$

of the fixed point set has an induced structure of a complex $G$-bundle. This property is fundamental for many calculations in geometric equivariant bordism theory.

We spelt out Definition 1 in order to avoid possible confusion with other variants of stable almost complex $G$-structures. For example, [6 contains the notion of a normally almost complex $G$-structure on a smooth $G$-manifold $M$, being defined as a complex $G$-bundle structure on the stable normal bundle of the embedding of $M$ in some real $G$-representation, see Definition 2.1 in loc. cit. The following example illustrates the difference between this notion and ours.

Let $S^{2}$ be equipped with the antipodal $\mathbb{Z} / 2$-action. Consider the $\mathbb{Z} / 2$ embedding

$$
S^{2} \subset \mathbb{R}^{3}
$$

where $\mathbb{Z} / 2$ acts on $\mathbb{R}^{3}$ by multiplication with -1 . The equivariant normal bundle of this embedding is

$$
\nu=S^{2} \times \mathbb{R} \rightarrow S^{2}
$$

with the trivial action on $\mathbb{R}$. Hence, $S^{2}$ is a normally almost complex $\mathbb{Z} / 2$-manifold in the sense of the above definition, although the quotient $S^{2} /(\mathbb{Z} / 2)$ is not even orientable. It is easy to check that $S^{2}$ equipped with this $\mathbb{Z} / 2$-action does not admit a stable almost complex $\mathbb{Z} / 2$-structure in the sense of Definition 1 Furthermore, we remark that for a free stable almost complex $G$-manifold $M$, the quotient $M / G$ always has an induced stable 
almost complex structure. From now on, we will exclusively work with Definition 1 .

For a $G$-space $X$, we define the geometric unitary $G$-equivariant bordism groups of $X$, denoted $\Omega_{n}^{G}(X)$, in the usual way as $G$-bordism classes of singular stable almost complex $G$-manifolds $M^{n} \rightarrow X$. The coefficients

$$
\Omega_{*}^{G}:=\Omega_{*}^{G}(\mathrm{pt} .)
$$

of this theory are equipped with a ring structure induced by the diagonal $G$-action on the cartesian product of two $G$-manifolds.

We recall the definition of homotopy theoretic unitary $G$-bordism introduced in [8]. Modern expositions of equivariant stable homotopy theory and $R O(G)$-graded homology theories can be found in [3, 12, 18]. For the sake of brevity, we restrict ourselves to an ad hoc definition of the relevant structures. Let

$$
\xi_{n}^{G} \rightarrow B U(n, G)
$$

be the universal unitary $n$-dimensional Grassmannian $G$-bundle and $T\left(\xi_{n}^{G}\right)$ its Thom space regarded as a pointed $G$-space.

Let $X$ be a pointed $G$-CW complex. By definition,

$$
\widetilde{M U}_{2 n}^{G}(X):=\lim _{W}\left[S^{W}, T\left(\xi_{|W|-n}^{G}\right) \wedge X\right]^{G}
$$

where $[-,-]^{G}$ is the group of homotopy classes of pointed $G$-equivariant maps and where the colimit is with respect to a directed set of unitary $G$ representations which ultimately contains each irreducible with arbitrary multiplicity. Recall ([12], Lemma (II.6.1)) that the suspension maps in the induced directed system of homotopy groups are uniquely determined, because we are using complex representations. Furthermore, we set

$$
\widetilde{M U}_{2 n-1}^{G}(X):=\widetilde{M U}_{2 n}^{G}\left(S^{1} \wedge X\right)
$$

where $S^{1}$ (with basepoint 1 ) carries the trivial $G$-action. Again, the coefficients

$$
M U_{*}^{G}:=\widetilde{M U}_{*}^{G}\left(S^{0}\right)
$$

carry a canonical ring structure.

The Pontrjagin-Thom map

$$
\Psi: \Omega_{*}^{G} \rightarrow M U_{*}^{G}
$$

is defined as follows: Let a stable almost complex $G$-manifold $M$ represent a bordism class in $\Omega_{2 n}^{G}$. We embed $M$ in a unitary $G$-representation $W$, and let $\nu$ be the normal bundle of this embedding. If $W$ is chosen large enough, the bundle $\nu$ carries an induced structure of a unitary $G$-bundle and we construct a map

$$
S^{W} \rightarrow T\left(\xi_{|\nu|}^{G}\right)
$$


from the universal bundle map $\nu \rightarrow \xi_{|\nu|}^{G}$ by collapsing the complement of the disk bundle $D(\nu)$ to the base point in $T\left(\xi_{|\nu|}^{G}\right)$. We explain in some detail how the required unitary $G$-bundle structure on $\nu$ is obtained. Let

$$
\iota: M \rightarrow V
$$

be a $G$-embedding into a unitary $G$-representation $V$ with (real) normal $G$ bundle $\mu$. We have fixed the structure of a unitary $G$-bundle on

$$
T M \oplus \underline{\mathbb{R}}^{2 k}
$$

for some $k$ (recall that we assume $\operatorname{dim} M$ to be even). Let $N$ be (the stably unique) complementary unitary $G$-bundle, i.e.

$$
\left(T M \oplus \underline{\mathbb{R}}^{2 k}\right) \oplus N=M \times U
$$

with a certain unitary $G$-representation $U$. The bundle $\mu \oplus \underline{U}$ is the normal bundle of the embedding

$$
M \rightarrow V \times U, x \mapsto(\iota(x), 0)
$$

and has an induced unitary $G$-structure, because

$$
\mu \oplus \underline{U}=\mu \oplus T M \oplus \underline{\mathbb{C}}^{k} \oplus N=\underline{V} \oplus \underline{\mathbb{C}}^{k} \oplus N .
$$

If $M$ is odd-dimensional, we obtain a map

$$
S^{W} \rightarrow S^{1} \wedge T\left(\xi_{|\widetilde{\nu}|}^{G}\right)
$$

because $\nu$ has a direct sum decomposition $\nu=\underline{\mathbb{R}} \oplus \widetilde{\nu}$ with a unitary $G$ bundle $\widetilde{\nu}$ (after choosing $W$ large enough).

The Pontrjagin-Thom map is a map of graded $M U_{*}$-algebras. We cite

Proposition 1 ([6], Theorem 5.4). For a compact abelian Lie group $G$, the Pontrjagin-Thom map is a split monomorphism of $M U_{*}$-modules.

A quick proof of the injectivity of $\Psi$ for the case $G=S^{1} \times \ldots \times S^{1}$ can be given, if one uses the fact (see [10], Theorem 3) that for topologically cyclic $G$, the characteristic number map

$$
\Omega_{*}^{G} \rightarrow K_{G}^{-*}\left[\left[a_{1}, a_{2}, \ldots\right]\right],
$$

which factors through the Pontrjagin-Thom map, is injective.

Let $W$ be a complex $G$-representation of dimension $n$ and let

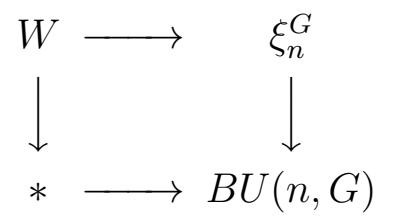

be the universal bundle map. The induced map $S^{0} \subset S^{W} \rightarrow T\left(\xi_{n}^{G}\right)$ represents the Euler class

$$
e_{W} \in M U_{-2 n}^{G}
$$


which is zero if and only if $W$ contains a trivial $G$-representation as a direct summand. In particular, for nontrivial $G$, the Pontrjagin-Thom map is not surjective, since the geometric theory $\Omega_{*}^{G}$ is concentrated in nonnegative degrees.

For dealing with the different $G$-representations we need a certain amount of bookkeeping device. We follow the exposition in [8]. Let $J$ be a set containing exactly one representative of each isomorphism class of nontrivial irreducible complex $G$-representations. We define the graded ring

$$
A_{*}(G):=\mathbb{Z}[\mathbb{Z} J]
$$

where $\mathbb{Z} J$ is the free abelian group generated by $J$. We consider $\mathbb{Z} J$ as a subgroup of the abelian group underlying the complex representation ring $R(G)$. The grading of $A_{*}(G)$ is induced by the real dimension of the underlying (virtual) $G$-representations. There is an isomorphism of graded rings

$$
A_{*}(G) \cong \mathbb{Z}\left[e_{V}, e_{V}^{-1}\right],
$$

where $V$ runs over elements in $J$ and each $e_{V}$ has degree $-2|V|$. This isomorphism is induced by the map

$$
\mathbb{Z} J \ni \sum_{V \in J} \alpha_{V} V \mapsto \prod e_{V}^{-\alpha_{V}}
$$

Elements in the ring $A_{*}(G)$ will be used in order to describe the (virtual) $G$-representations occuring around the fixed set of a given element in $M U_{*}^{G}$.

Fix a basepoint 1 in $B U$ and let

$$
B \subset B U^{J}
$$

be the subset consisting of $J$-indexed families in $B U$ of which only finitely many components are different from the base point. Whitney sum of vector bundles induces an $H$-space structure

$$
m: B U \times B U \rightarrow B U .
$$

We can assume that $m(1,1)=1$. With the componentwise multiplication, we get an $H$-space structure on $B$. The space $B$ is the classifying space for unitary $G$-bundles over base spaces $X$ with trivial $G$-action and with $G$ acting without fixed points in each fibre. More precisely, the $V$-th component of a map

$$
X \rightarrow B
$$

classifies the $V$-isotypical component $E_{V}$ of a given such $G$-bundle

$$
\bigoplus_{V \in J} E_{V} \otimes V \rightarrow X .
$$


In [8], p. 350, tom Dieck constructs a map of graded $M U_{*}$-algebras

$$
\phi_{M U}:=\phi: M U_{*}^{G} \rightarrow M U_{*}(B) \otimes A_{*}(G) .
$$

Heuristically, this map is given by restriction to fixed point sets. This becomes especially clear in the slightly different description of the map $\phi$ in [21], which we now briefly recall (for a more detailed exposition, see [21]): Let

$$
f: S^{W} \rightarrow T\left(\xi_{n}^{G}\right)
$$

represent an element $c \in M U_{2(|W|-n)}^{G}$. Consider the restriction

$$
f^{G}:\left(S^{W}\right)^{G} \rightarrow T\left(\xi_{n}^{G}\right)^{G}
$$

of $f$ to fixed point sets. This map represents an element in

$$
\left(\phi^{G} M U^{G}\right)_{2(|W|-n)}
$$

where $\phi^{G} M U^{G}$ is the geometric fixed points spectrum associated with the equivariant unitary bordism spectrum $M U^{G}$. We have an equivalence of ring spectra

$$
\phi^{G} M U^{G} \simeq \mathbf{I}_{R(G)} \wedge M U \wedge B_{+}
$$

where $M U$ is the usual unitary bordism spectrum and

$$
\mathbf{I}_{R(G)}:=\bigvee_{W \in R(G),|W|=0} S^{2\left|W^{G}\right|}
$$

is the one point union of suspended sphere spectra equipped with a ring spectrum structure induced by addition of elements in $R(G)$, cf. Theorem 4.9. in [21]. We consider the stable homotopy of $\mathbf{I}_{R(G)} \wedge M U \wedge B_{+}$as a collection of suspended copies of $M U_{*}(B)$ and hence get an isomorphism of graded rings

$$
\omega:\left(\mathbf{I}_{R(G)} \wedge M U \wedge B_{+}\right)_{*} \cong M U_{*}(B) \otimes A_{*}(G) .
$$

It is induced by identifying the summand of $M U_{*}(B)$ indexed by $W \in$ $R(G)$ with

$$
M U_{*}(B) \otimes\left(e_{\left(U^{G}\right)^{\perp}} \cdot\left(e_{\left(V^{G}\right)^{\perp}}^{-1}\right)\right) \subset M U_{*}(B) \otimes A_{*}(G) .
$$

Here, we write $W=U-V$ with unitary $G$-representations $U$ and $V$ and use splittings $U=U^{G} \oplus\left(U^{G}\right)^{\perp}$ and $V=V^{G} \oplus\left(V^{G}\right)^{\perp}$. Finally, we set

$$
\phi(c):=\omega\left(\left[f^{G}\right]\right) .
$$

For elements in $M U_{*}^{G}$ of odd degree, the definition of $\phi$ is similar. Note the tautological equation

$$
\phi_{M U}\left(e_{V}\right)=e_{V}
$$

for each nontrivial irreducible $G$-representation $V$. 
The Künneth formula shows that for each finite subset $J^{\prime} \subset J$, we have

$$
M U_{*}\left(\prod_{V \in J^{\prime}} B U\right) \cong \bigotimes_{V \in J^{\prime}} M U_{*}(B U) \text {. }
$$

where the tensor product is over $M U_{*}$. By a standard application of the Atiyah-Hirzebruch spectral sequence,

$$
M U_{*}(B U) \cong M U_{*}\left[X_{1}, X_{2}, X_{3}, \ldots\right]
$$

is a polynomial algebra over $M U_{*}$ in infinitely many indeterminates $X_{d}$ of degree $2 d, 1 \leq d<\infty$, see [14]. We can and will choose the polynomial generators $X_{d}$ as being represented by

$$
\mathbb{C} P^{d} \longrightarrow B U
$$

classifying the hyperplane line bundle over $\mathbb{C} P^{d}$. Altogether (using that $M U_{*}$ commutes with direct limits), we get an isomorphism of graded $M U_{*}$ algebras

$$
M U_{*}(B) \otimes A_{*}(G) \cong M U_{*}\left[e_{V}, e_{V}^{-1}, Y_{V, d}\right]
$$

where $V$ runs over the nontrivial irreducible $G$-representations and $Y_{V, d}$, $|V|+1 \leq d<\infty$, is the image of the element

$$
X_{d-|V|} \otimes e_{V}^{-1} \in M U_{2 d-2|V|}(B U) \otimes A_{2|V|}(G)
$$

under the inclusion map $B U \rightarrow B$ which embeds $B U$ as the $V$ th factor. If we had defined $X_{0}$ as the unit in $M U_{0}(B U)$, the corresponding element $Y_{V,|V|}$ would be equal to $e_{V}^{-1}$.

Heuristically, for a geometric class $[M] \in \Omega_{*}^{G}$, the element

$$
\phi_{M U} \circ \Psi([M]) \in M U_{*}\left[e_{V}, e_{V}^{-1}, Y_{V, d}\right]
$$

describes the stable normal bundle of $M$ restricted to $M^{G}$. In particular, for each one-dimensional nontrivial irreducible complex $G$-representation $V$, we have

$$
\iota \circ \phi_{M U} \circ \Psi\left(\left[\mathrm{P}\left(\mathbb{C}^{d} \oplus V\right)\right]\right)=Y_{V, d}+e_{V^{*}}^{-d},
$$

where $V^{*}$ is the conjugate representation, $P$ denotes the projectivization and

$$
\iota: M U_{*}\left[e_{V}, e_{V}^{-1}, Y_{V, d}\right] \rightarrow M U_{*}\left[e_{V}, e_{V}^{-1}, Y_{V, d}\right]
$$

is induced by the inverse of the $H$-space $B$ (we identify $M U_{*}(B) \otimes A_{*}(G)$ and $M U_{*}\left[e_{V}, e_{V}^{-1}, Y_{V, d}\right]$ by the isomorphism constructed before). The map $\iota$ interchanges the roles of the stable normal bundle of $M$ restricted to $M^{G}$ and of the normal bundle of $M^{G}$ in $M$. Note that $\iota \circ \Phi_{M U}\left(e_{V}\right)=e_{V}$ for all $V$. The above equation can be checked by inspecting the normal bundle of $\mathrm{P}\left(\mathbb{C}^{d} \oplus V\right)^{G}$ in $\mathrm{P}\left(\mathbb{C}^{d} \oplus V\right)$, cf. Prop. 4.14. in [21] (in this reference, the map $\iota$ is mistakenly left out). 
We now discuss the geometric analogue of the map $\phi_{M U}$ and define a map of $M U_{*}$-algebras

$$
\phi_{\Omega}: \Omega_{*}^{G} \rightarrow M U_{*}(B) \otimes A_{*}(G)
$$

in the following way: Let $M^{n}$ be a stable almost complex $G$-manifold and let

$$
F \subset M^{G}
$$

be a connected component of the fixed point set. The normal bundle $\nu(F)$ of $F$ in $M$ is a complex $G$-bundle in a canonical way. Let $k$ be its complex dimension. Write

$$
\nu(F)=\left(E_{1} \otimes V_{1}\right) \oplus \ldots \oplus\left(E_{j} \otimes V_{j}\right)
$$

with complex vector bundles

$$
E_{1}, \ldots, E_{j}
$$

and irreducible $G$-representations

$$
V_{1}, \ldots, V_{j}
$$

Now define

$$
b_{F}:=\bar{b}_{F} \otimes\left(e_{V_{1}}^{-\left|E_{1}\right|} \cdot \ldots \cdot e_{V_{j}}^{-\left|E_{j}\right|}\right) \in M U_{n-2 k}(B) \otimes A_{2 k}(G)
$$

where $\bar{b}_{F} \in M U_{n-2 k}(B)$ is represented by the map

$$
F \rightarrow B
$$

with the $V_{i}$-th component classifying the bundle $E_{i}$. Finally, we set

$$
\phi_{\Omega}([M]):=\sum_{F \subset M^{G}} b_{F} \in(M U(B) \otimes A(G))_{n} .
$$

The following result of tom Dieck shows that the geometric and homotopy theoretic fixed point maps are compatible with respect to the PontrjaginThom map.

Proposition 2 ([[8], Proposition 4.1). The following diagram is commutative.

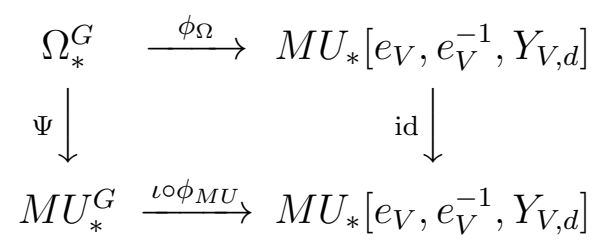




\section{GEOMETRIC REALIZABILITY}

Definition 2. We define the geometric cone in $M U_{*}\left[e_{V}, e_{V}^{-1}, Y_{V, d}\right]$ as the $M U_{*}$-subalgebra

$$
\Gamma_{*}:=M U_{*}\left[e_{V}^{-1}, Y_{V, d}\right] .
$$

The following proposition justifies this name.

Proposition 3. $\phi_{\Omega}\left(\Omega_{*}^{G}\right) \subset \Gamma_{*}$.

Proof. Due to the definition of the polynomial generators $Y_{V, d}$, the proof is nontrivial. Let $M$ be a stable almost complex $G$-manifold and let

$$
F \subset M^{G}
$$

be a fixed point component with normal bundle $\nu(F)$ of complex dimension $k$. As above, we write

$$
\nu(F)=\left(E_{1} \otimes V_{1}\right) \oplus \ldots \oplus\left(E_{j} \otimes V_{j}\right) .
$$

We show that the element

$$
\bar{b}_{F} \in M U_{n-2 k}(B) \otimes \mathbb{Z} \subset M U_{n-2 k}(B) \otimes A_{2 k}(G)=M U_{*}\left[e_{V}, e_{V}^{-1}, Y_{V, d}\right]
$$

is a polynomial in $e_{V_{i}}$ of degree at most $\left|E_{i}\right|$ for all $V_{i} \in\left\{V_{1}, \ldots, V_{j}\right\}$. This in turn implies that

$$
b_{F}:=\bar{b}_{F} \otimes\left(e_{V_{1}}^{-\left|E_{1}\right|} \cdot \ldots \cdot e_{V_{j}}^{-\left|E_{j}\right|}\right) \in M U_{n-2 k}(B) \otimes A_{2 k}(G)
$$

is indeed an element of $M U_{*}\left[e_{V}^{-1}, Y_{V, d}\right]$.

The bordism class $\bar{b}_{F}$ can be regarded as being represented by a map

$$
F \rightarrow B U\left(\left|E_{1}\right|\right) \times \ldots \times B U\left(\left|E_{j}\right|\right) .
$$

Each element in $M U_{*}\left(B U\left(\left|E_{i}\right|\right)\right), 1 \leq i \leq j$, can be written as a sum of monomials in the $X_{d}$ 's each of which contains at most $\left|E_{i}\right|$ factors of the form $X_{d}$, see e.g. [14], Proposition 4.3.3 a). This implies that under the change of variables sending $X_{d}$ to $Y_{V_{i}, d+\left|V_{i}\right|} \otimes e_{V_{i}}$, each such element is expressible as a polynomial in $M U_{*}\left[e_{V_{i}}, Y_{V_{i}, d}\right]$ of degree at most $\left|E_{i}\right|$ in $e_{V_{i}}$. Hence, using the Künneth formula for computing

$$
M U_{*}\left(B U\left(\left|E_{1}\right|\right) \times \ldots \times B U\left(\left|E_{j}\right|\right)\right)
$$

the element $\bar{b}_{F}$ is indeed of the required form. This finishes the proof of Proposition 3 . 
Theorem 1. Let $G=S^{1} \times \ldots \times S^{1}$. Then the commutative diagram of $M U_{*}$-algebras

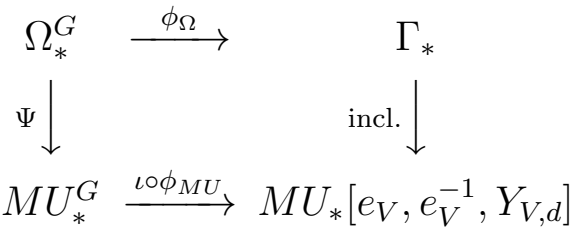

induced by the commutative fixed point square of tom Dieck (see Proposition (2) is a pull back square. All maps in this diagram are injective.

For $G$ a torus, the map $\phi_{M U}$ is injective by [21], Proposition 4.5 and Corollary 5.2. Thus (together with Proposition 1) only the pull back property requires proof.

The last theorem implies the following relation of geometric and homotopy theoretic equivariant bordism.

Corollary 1. Let $G$ be a torus. Then the Pontrjagin-Thom map induces an isomorphism of $M U_{*}$-algebras

$$
\Omega_{*}^{G} \cong \operatorname{im}\left(\iota \circ \phi_{M U}\right) \cap \Gamma_{*} .
$$

Hence, the coefficients of the geometric equivariant $\left(S^{1}\right)^{r}$-bordism ring constitute a certain subring of $M U_{*}\left[e_{V}, e_{V}^{-1}, Y_{V, d}\right]$ whose elements are characterized by the following two conditions: Firstly, they must lie in the image of $\iota \circ \phi_{M U}$. Heuristically, the local fixed point data specified by an element in $M U_{*}\left[e_{V}, e_{V}^{-1}, Y_{V, d}\right]$ can be "closed up" (without introducing new fixed points) such as to define an actual class in $M U_{*}^{G}$. This property can be formalized using bordism with respect to families of subgroups of $G$ (see below). Secondly, they must lie in the geometric cone.

The first of these two conditions has been extensively studied by tom Dieck [8, 11] in terms of integrality conditions related to the localization techniques of Atiyah-Segal. We will come back to this description in Section 5 .

We now turn to the proof of Theorem 1. Recall that a family of subgroups $\mathcal{F}$ of a topological group $G$ is a set of closed subgroups of $G$ which is closed under conjugation and under taking subgroups. Such a family is supposed to encode the possible isotropy groups occuring in a given $G$-space. We define special families of subgroups of $G$ :

$$
\begin{aligned}
& \mathcal{A}:=\{H<G\}, \\
& \mathcal{P}:=\{H<G \mid H \neq G\} .
\end{aligned}
$$

From now on, we assume that $G$ is a compact Lie group. For each family of subgroups $\mathcal{F}$, there exists a classifying space $E \mathcal{F}$ [12], I.(6.6), which is a terminal object in the homotopy category of $\mathcal{F}$-numerable $G$-spaces. The space $E \mathcal{F}$ is characterized by the properties 
- $E \mathcal{F}^{H} \simeq *$, if $H \in \mathcal{F}$,

- $E \mathcal{F}^{H}=\emptyset$, if $H \notin \mathcal{F}$.

(using nonequivariant homotopy equivalences). Furthermore, it is unique up to $G$-homotopy equivalence. For a $G$-space $X$ and a pair of families of subgroups $\left(\mathcal{F}, \mathcal{F}^{\prime}\right)$ (i.e. $\mathcal{F}^{\prime} \subset \mathcal{F}$ ), we set

$$
\begin{aligned}
\Omega_{n}^{G}\left[\mathcal{F}, \mathcal{F}^{\prime}\right](X) & :=\Omega_{n}^{G}\left(X \times E \mathcal{F}, X \times E \mathcal{F}^{\prime}\right), \\
M U_{n}^{G}\left[\mathcal{F}, \mathcal{F}^{\prime}\right](X) & :=M U_{n}^{G}\left(X \times E \mathcal{F}, X \times E \mathcal{F}^{\prime}\right) .
\end{aligned}
$$

cf. [6], p. 339. The groups $\Omega_{n}^{G}\left[\mathcal{F}, \mathcal{F}^{\prime}\right](X)$ consist of $G$-bordism classes of stable almost complex $G$-manifolds $\left(M^{n}, \partial M\right)$ with boundary and with reference maps to $X$ such that all isotropy groups occuring in $M$ (resp. in $\partial M$ ) lie in $\mathcal{F}$ (resp. in $\mathcal{F}^{\prime}$ ). The long exact sequence of the pair

$$
\left(X \times E \mathcal{F}, X \times E \mathcal{F}^{\prime}\right)
$$

is the Conner-Floyd exact sequence

$$
\ldots \rightarrow \Omega_{n}^{G}\left[\mathcal{F}^{\prime}\right](X) \rightarrow \Omega_{n}^{G}[\mathcal{F}](X) \rightarrow \Omega_{n}^{G}\left[\mathcal{F}, \mathcal{F}^{\prime}\right](X) \rightarrow \Omega_{n-1}^{G}\left[\mathcal{F}^{\prime}\right](X) \rightarrow \ldots
$$

The proof of Theorem 1 is based on studying the commutative diagram with exact rows

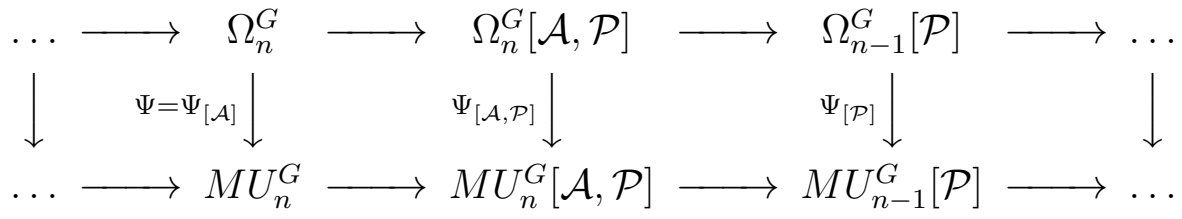

whose vertical arrows are Pontrjagin-Thom maps. We know from [6], Theorem 5.4, that $\Psi_{[\mathcal{A}]}$ is injective. In a first step, we will identify the terms occuring in the second column by showing the existence of a commutative diagram

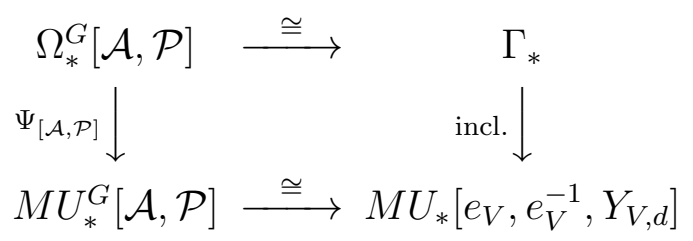

such that the compositions of the horizontal maps with the canonical maps

$$
\Omega_{n}^{G} \rightarrow \Omega_{n}^{G}[\mathcal{A}, \mathcal{P}], M U_{n}^{G} \rightarrow M U_{n}^{G}[\mathcal{A}, \mathcal{P}]
$$

coincide with the fixed point maps $\phi_{\Omega}$ and $\iota \circ \phi_{M U}$. This will be done in Proposition 4 In a second step we show in Proposition [5] that the map $\Psi_{[\mathcal{P}]}$ is injective. For this, we use equivariant characteristic numbers as introduced by tom Dieck [9, 10]. From these facts together with the injectivity of $\Psi_{[\mathcal{A}]}$ and the injectivity of the fixed point map $\iota \circ \phi_{M U}$, the assertion of Theorem 1 follows by an easy diagram chase. 
Proposition 4. Let $G$ be a compact Lie group. Then there are ring isomorphisms

$$
\kappa_{\Omega}: \Omega_{*}^{G}[\mathcal{A}, \mathcal{P}] \rightarrow \Gamma_{*}
$$

and

$$
\kappa_{M U}: M U_{*}^{G}[\mathcal{A}, \mathcal{P}] \rightarrow M U_{*}\left[e_{V}, e_{V}^{-1}, Y_{V, d}\right]
$$

such that the diagram

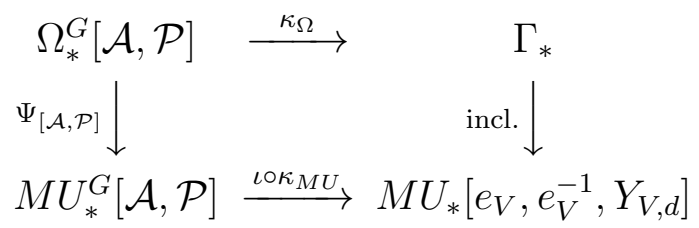

is commutative and the compositions

$$
\Omega_{*}^{G} \rightarrow \Omega_{*}^{G}[\mathcal{A}, \mathcal{P}] \stackrel{\kappa_{\Omega}}{\longrightarrow} \Gamma_{*}
$$

and

$$
M U_{*}^{G} \rightarrow M U_{*}^{G}[\mathcal{A}, \mathcal{P}] \stackrel{\kappa_{M U}}{\longrightarrow} M U_{*}^{G}\left[e_{V}, e_{V}^{-1}, Y_{V, d}\right]
$$

coincide with the fixed point maps $\phi_{\Omega}$ and $\phi_{M U}$, respectively.

Proof. We construct a map

$$
\kappa_{\Omega}: \Omega_{*}^{G}[\mathcal{A}, \mathcal{P}] \rightarrow \Gamma_{*} \subset M U_{*}(B) \otimes A_{*}(G)
$$

as follows. The group $\Omega_{n}^{G}[\mathcal{A}, \mathcal{P}]$ is generated by elements represented by (disc bundles of) unitary $G$-bundles

$$
E \rightarrow M
$$

where $M$ is a stable almost complex manifold of dimension $n-2 k$ with trivial $G$-action, $E$ has complex fibre dimension $k$ and $E^{G}=M$. We define the image of $[E \rightarrow M]$ under $\kappa_{\Omega}$ in exactly the same way as we defined the geometric fixed point map $\phi_{\Omega}$ above. The proof of the fact that we get indeed an element in $\Gamma_{*}$ is similar as before. An inverse of $\kappa_{\Omega}$ is constructed as follows. Let $V$ be a nontrivial irreducible unitary $G$-representation. We map $Y_{V, d}$ to the disc bundle of the $G$-bundle

$$
E \otimes V \rightarrow \mathbb{C} P^{d-|V|}
$$

where $E \rightarrow \mathbb{C} P^{d-|V|}$ is the hyperplane line bundle. The element $e_{V}^{-1}$ is mapped to the disc bundle of the trivial $G$-bundle

$$
V \rightarrow * \text {. }
$$

Now $\kappa_{\Omega}^{-1}$ is determined by the fact that it is a map of $M U_{*}$-algebras.

On the homotopy theoretic side, the construction is as follows. The map

$$
\kappa_{M U}: M U_{*}^{G}[\mathcal{A}, \mathcal{P}] \rightarrow M U_{*}\left[e_{V}, e_{V}^{-1}, Y_{V, d}\right]
$$


is defined as the composition

$$
M U_{*}^{G}[\mathcal{A}, \mathcal{P}] \cong \widetilde{M U}_{*}^{G}(\Sigma E \mathcal{P}) \cong\left(\phi^{G} M U^{G}\right)_{*} \cong M U_{*}\left[e_{V}, e_{V}^{-1}, Y_{V, d}\right] .
$$

In this sequence, the first isomorphism is induced by the excisive inclusion of $G$-spaces

$$
(E \mathcal{A}, E \mathcal{P}) \simeq\left(C_{-} E \mathcal{P}, E \mathcal{P}\right) \rightarrow\left(\Sigma E \mathcal{P}, C_{+} E \mathcal{P}\right)
$$

where $C_{-}$and $C_{+}$denote the lower and upper cone in the unreduced suspension

$$
\Sigma E \mathcal{P}=([0,1] \times E \mathcal{P}) / \sim
$$

which we consider as being equipped with the basepoint $[\{1\} \times E \mathcal{P}]$ and as containing $E \mathcal{P}$ as the subspace $\{1 / 2\} \times E \mathcal{P}$. The second isomorphism is induced by Lemma 4.2. in [21] with $Z:=\Sigma E \mathcal{P}$ and the third isomorphism is Theorem 4.10. in loc. cit.

The commutativity of the diagram in Proposition 4 is proven in a similar fashion as in the case of tom Dieck's fixed point square (cf. Proposition 2). We omit the details. The remaining assertions are immediate.

We now deal with the second step of the proof of Theorem 1 and show the following variant of [6], Theorem 5.4.

Proposition 5. Let $G$ be topologically cyclic group, i.e. $G$ is of the form $S^{1} \times \ldots \times S^{1} \times \mathbb{Z} / k, k=1,2, \ldots$. Then the Pontrjagin-Thom map

$$
\Psi_{[\mathcal{P}]}: \Omega_{n}^{G}[\mathcal{P}] \rightarrow M U_{n}^{G}[\mathcal{P}]
$$

is injective for all $n$.

Proof. If $G=S^{1}$, the assertion is implied by [6], Theorem 7.1.(3) with the choice $G:=\{e\}$. The general case can conveniently be dealt with using equivariant characteristic numbers [9, 10]. It follows from [10], Theorem 3 , that for topologically cyclic $G$ and a unitary $G$-representation $W$, the characteristic number map

$$
\Omega_{*}^{G}(S W) \rightarrow K_{G}^{-*}(S W)\left[\left[a_{1}, a_{2}, \ldots\right]\right]
$$

is injective. Here, $S W$ denotes the unit sphere in $W$. But this map factors through the Pontrjagin-Thom map

$$
\Omega_{*}^{G}(S W) \rightarrow M U_{*}^{G}(S W) .
$$

Now observe that

$$
E \mathcal{P}=\lim _{\longrightarrow} S W
$$


where the colimit is taken with respect to a directed set of complex $G$ modules without trivial direct summands and so that ultimately each nontrivial irreducible appears with arbitrarily large multiplicity in some $G$ module $W$. Finally, the claim of Proposition 5 follows because homology commutes with direct limits:

$$
\begin{aligned}
\Omega_{*}^{G}(E \mathcal{P}) & =\lim _{W} \Omega_{*}^{G}(S W), \\
M U_{*}^{G}(E \mathcal{P}) & =\lim _{W} \Omega_{*}^{G}(S W) .
\end{aligned}
$$

We remark that Proposition 5 holds for any compact abelian Lie group $G$ and with $\mathcal{P}$ replaced by any family of subgroups of $G$. The proof of this general statement can be carried out using similar ideas as in [6] and is based on a systematic use of the Conner-Floyd exact sequences for bordism with respect to families of subgroups. However, it is considerably more tedious than the proof presented above for topologically cyclic $G$. Since only this case is of importance for us, we decided not to include the more general case in this paper.

\section{THE CASE OF NONCONNECTED $G$}

There are simple examples showing that if $G$ is not connected and not of prime order, then the geometric realizability of an element in $M U_{*}^{G}$ is not determined by the normal data around fixed point sets. Let $n$ be a natural number, $G=\mathbb{Z} / n \times \mathbb{Z} / n$ and let

$$
f: \mathbb{Z} / n \rightarrow B U(1, G)
$$

classify the bundle

$$
\mathbb{Z} / n \times V \rightarrow \mathbb{Z} / n
$$

where $V$ is the canonical complex 1-dimensional representation of $\mathbb{Z} / n$, the space $\mathbb{Z} / n \times V$ carries the product $(\mathbb{Z} / n \times \mathbb{Z} / n)$-action and the bundle map is simply projection onto the first factor. The map

$$
\mathbb{Z} / n \times S^{0} \subset \mathbb{Z} / n \times S^{V} \rightarrow T\left(\xi_{1}^{G}\right)
$$

induced by $f$ represents an element $c \in M U_{-2}^{G}$ which is different from zero, because its restriction to $(\{1\} \times \mathbb{Z} / n)$-bordism is $n$ times the Euler class $e_{V} \in M U_{-2}^{\mathbb{Z} / n}$ (this is known to be a nontorsion class). However,

$$
\phi_{M U}(c)=0 .
$$

A similar example exists for $G=\mathbb{Z} / n^{2}$ with $f: \mathbb{Z} / n \rightarrow B U(1, G)$ classifying the bundle

$$
\mathbb{Z} / n \times W \rightarrow \mathbb{Z} / n
$$


where $W$ is the canonical one dimensional $\mathbb{Z} / n^{2}$-representation and $G$ acts on the $\mathbb{Z} / n$-factors via the projection $G \rightarrow \mathbb{Z} / n$ with kernel $\mathbb{Z} / n$. The restriction of the corresponding bordism class to $\mathbb{Z} / n$-bordism (where $\mathbb{Z} / n \subset$ $G$ is of index $n$ ), is again given as $n$ times the Euler class $e_{V} \in M U_{-2}^{\mathbb{Z} / n}$.

One reason of the failure of Theorem 1 for arbitrary compact abelian $G$ is the fact that the fixed point map

$$
\phi_{M U}: M U_{*}^{G} \rightarrow M U_{*}\left[e_{V}, e_{V}^{-1}, Y_{V, d}\right]
$$

is injective if and only if $G$ is a torus, see [21], Theorem 5.1. It seems plausible that for geometric realizability of an element in $M U_{*}^{G}$ for general $G$, a series of obstructions must vanish each one of which lies in some group

$$
M U_{*}^{G}\left[\mathcal{F}, \mathcal{F}^{\prime}\right]
$$

with an adjacent pair of families $\left(\mathcal{F}, \mathcal{F}^{\prime}\right)$ and each one of which is only defined if the previous one vanishes. However, we do not know a concise statement in this direction.

\section{INTEGRALITY}

We will study the image of the map

$$
M U_{*}^{G} \rightarrow M U_{*}^{G}[\mathcal{A}, \mathcal{P}] \stackrel{\left\llcorner\circ \kappa_{M U}\right.}{\cong} M U_{*}\left[e_{V}, e_{V}^{-1}, Y_{V, d}\right]
$$

(which occurs in the lower line of the diagram in Theorem 1) in the light of integrality conditions formulated by tom Dieck. We have a natural multiplicative transformation

$$
\eta: M U_{G}^{*}(X) \rightarrow M U^{*}\left(E G \times_{G} X\right)
$$

where $X$ is an arbitrary $G$-CW complex. This map (called "bundling transformation" in the papers of tom Dieck) is induced by the projection

$$
E G \times X \rightarrow X
$$

yielding a map

$$
M U_{G}^{*}(X) \rightarrow M U_{G}^{*}(E G \times X) \cong M U^{*}\left(E G \times_{G} X\right) .
$$

For a complex $G$-representation $W$, let $e(W) \in M U^{2}(B G)$ denote the bordism theoretic Euler class of the complex vector bundle

$$
E G \times_{G} W \rightarrow B G .
$$

It follows directly from the definition of $\eta$ that

$$
\eta\left(e_{W}\right)=e(W) .
$$

Here, we regard $e_{W}$ as an element in $M U_{G}^{2|W|}=M U_{-2|W|}^{G}$. Recall that there is an isomorphism

$$
M U^{*}\left(B\left(S^{1}\right)^{r}\right) \cong M U^{*}\left[\left[C_{1}, \ldots, C_{r}\right]\right]
$$


where $C_{i} \in M U^{2}\left(B\left(S^{1}\right)^{r}\right)$ is the Euler class of the $\left(S^{1}\right)^{r}$-representation $\rho_{i}$ which is induced from the standard one-dimensional $S^{1}$-representation by projecting $\left(S^{1}\right)^{r}$ onto the $i$ th factor. If

$$
V=\rho_{1}^{\otimes \mu_{1}} \otimes \ldots \otimes \rho_{r}^{\otimes \mu_{r}}
$$

then

$$
e(V)=\left[\mu_{1}\right]_{F} C_{1}+{ }_{F} \ldots+{ }_{F}\left[\mu_{r}\right]_{F} C_{r}
$$

with Lazard's universal formal group law $+_{F}$ in $M U^{*}\left[\left[C_{1}, \ldots, C_{r}\right]\right]$.

Let $S \subset M U_{G}^{*}=M U_{-*}^{G}$ denote the multiplicative subset of Euler classes of $G$-representations without trivial direct summand. We denote the corresponding subset of $M U^{*}(B G)$ by $S$ as well and obtain an induced map

$$
S^{-1} \eta: M U_{*}^{G}[\mathcal{A}, \mathcal{P}] \cong S^{-1} M U_{*}^{G} \rightarrow S^{-1} M U^{-*}(B G)
$$

compatible with $\eta$ in the obvious way. The following result of tom Dieck [10] characterizes the image of

$$
\iota \circ \Phi_{M U}: M U_{*}^{G} \rightarrow M U_{*}^{G}[\mathcal{A}, \mathcal{P}] \stackrel{\iota \circ \kappa_{M U}}{\cong} M U_{*}\left[e_{V}, e_{V}^{-1}, Y_{V, d}\right] .
$$

We will regard $S^{-1} \eta$ as being defined on $M U_{*}\left[e_{V}, e_{V}^{-1}, Y_{V, d}\right]$ by identifying this module with $M U_{*}^{G}[\mathcal{A}, \mathcal{P}]$ using the isomorphism $\iota \circ \kappa_{M U}$.

Proposition $6([10])$. Let $G$ be a topologically cyclic group and let

$$
\lambda: M U^{*}(B G) \rightarrow S^{-1} M U^{*}(B G)
$$

be the localization map. Then

$$
\operatorname{im}\left(\iota \circ \Phi_{M U}\right)=\left\{x \in M U_{*}\left[e_{V}, e_{V}^{-1}, Y_{V, d}\right] \mid S^{-1} \eta(x) \in \lambda\left(M U^{-*}(B G)\right)\right\}
$$

For $G=S^{1} \times \ldots \times S^{1}$, the localization map $\lambda$ is injective, because $M U^{*}(B G)$ has no zero divisors. Therefore, $M U^{*}\left[\left[C_{1}, \ldots, C_{r}\right]\right]$ can be considered as a subring of its $S$-localization. In combination with Theorem 1 and by the fact that for toral $G$ the map $\eta$ is injective (because the characteristic number map factors through $\eta$ ), we now get the following description of the geometric unitary $G$-bordism ring.

Theorem 2. Let $G=\left(S^{1}\right)^{r}$. Let

$$
\text { incl. : } M U_{*}\left[e_{V}^{-1}, Y_{V, d}\right] \rightarrow M U_{*}\left[e_{V}, e_{V}^{-1}, Y_{V, d}\right]
$$

be the canonical inclusion. As before, we identify $M U_{n}$ and $M U^{-n}$. Then the diagram

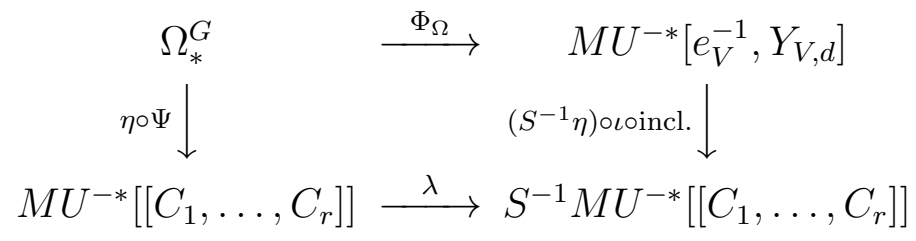

is a pull back square of $M U_{*}$-algebras. 
Acknowledgements: The author is grateful to U. Bunke and T. Schick for helpful comments.

\section{REFERENCES}

[1] M. Atiyah, G. Segal: The index of elliptic operators: II. Ann. Math. 87, (1968) 531545 .

[2] T. Bröcker, T. Hook: Stable equivariant bordism. Math. Z. 129, (1972) 269-277.

[3] G. Carlsson: A survey of equivariant stable homotopy theory. Topology 31 (1), (1992) $1-27$.

[4] M. Cole, J. Greenlees, I. Kriz: Equivariant formal group laws. Proc. London Math. Soc. (3) 81, (2000) 355-386.

[5] M. Cole, J. Greenlees, I. Kriz: The universality of equivariant complex bordism. Math. Z. 239, (2002) 455-475.

[6] G. Comezaña: Calculations in complex equivariant bordism. In: J. P. May (ed.): Equivariant Homotopy and Cohomology theory, CBMS Regional Conference Series in Mathematics 90, (1996) 333-352.

[7] P. Conner, E. Floyd: Differentiable periodic maps. Ergebnisse der Mathematik und ihrer Grenzgebiete, Band 33, Springer-Verlag (1964).

[8] T. tom Dieck: Bordism of $G$-manifolds and integrality theorems. Topology 9, (1970) 345-358.

[9] T. tom Dieck: Characteristic numbers of $G$-manifolds I. Invent. Math. 13, (1971) 213224.

[10] T. tom Dieck: Characteristic numbers of $G$-manifolds II. Journal of pure and applied algebra 4, (1974) 31-39.

[11] T. tom Dieck: Periodische Abbildungen unitärer Mannigfaltigkeiten. Math. Z. 126, (1972) 275-295.

[12] T. tom Dieck: Transformation groups. De Gruyter Studies in Mathematics 8, (1987).

[13] J. Greenlees, P. May: Localization and completion theorems for $M U$-module spectra. Ann. Math. 146, (1997) 509-544.

[14] S. O. Kochman: Bordism, Stable homotopy and Adams spectral sequences. Fields institute monographs, AMS (1996).

[15] C. Kosniowski: Generators of the $\mathbb{Z} / p$-bordism ring. Serendipity. Math. Z. 149, (1976) 121-130.

[16] C. Kosniowski, M. Yahia: Unitary bordism of circle actions. Proc. Edinburgh Math. Soc. 26, (1983) 97-105.

[17] I. Kriz: The $\mathbb{Z} / p$-equivariant complex bordism ring. Contemp. Math. 239, (1999) 217-223.

[18] L. Lewis Jr., J. May and M. Steinberger: Equivariant stable homotopy theory. LNM 1213, Springer Verlag (1985).

[19] P. Löffler: Equivariant unitary cobordism and classifying spaces. Proceedings of the International Symposium on Topology and its Applications (Budva, 1972), 158-160.

[20] T. Petrie: $G$-transversality. Bull. AMS 81 (4), (1975) 721-722.

[21] D. Sinha: Computations of complex equivariant bordism rings. American Journal of Mathematics 123, (2001) 577-605.

[22] D. Sinha: Bordism of semifree $S^{1}$-actions. Math. Z. 249, (2005) 439-454. 